\title{
Epicardial adipose tissue and peripheral artery disease: Protocol for systematic review and meta-analysis
}

Leonardo Roever $^{1 *}$, Elmiro Santos Resende ${ }^{1}$, Angélica LemosDebs Diniz ${ }^{1}$, Nilson Penha-Silva ${ }^{1}$, João Lucas O'Connell ${ }^{1}$, Fernanda Rodrigues de Souza $^{1}$, Poliana Rodrigues Alves Duarte ${ }^{1}$, Paulo Fernando Silva Gomes ${ }^{1}$, Hugo Ribeiro Zanetti ${ }^{2}$, Anaisa Silva Roerver-Borges ${ }^{2}$, Fernando César Veloso $^{1}$, Thiago Montes Fidale ${ }^{1}$, Antonio Casella-Filho ${ }^{3}$, Paulo Magno Martins Dourado ${ }^{3}$, Antonio Carlos Palandri Chagas ${ }^{3,4}$, Sadeq Ali-Hasan-Al-Saegh ${ }^{5}$, Paulo Eduardo Ocke Reis ${ }^{6}$, Priscila Pollo-Flores ${ }^{6}$, Rogério de MeloCosta Pinto ${ }^{1}$, Gustavo BF Oliveira ${ }^{7}$, Álvaro Avezum, Mansueto Neto ${ }^{8}$, André Durães ${ }^{8}$, Rose Mary Ferreira Lisboa da Silva ${ }^{9}$, Antonio José Grande ${ }^{10}$, Celise Denardi ${ }^{11}$, Renato Delascio Lopes $^{12}$, Nitesh Nerlekar ${ }^{13}$, Shahab Alizadeh ${ }^{14}$, Adrian V Hernandez ${ }^{15}$, Maria Inês da Rosa ${ }^{16}$, Gary Tse ${ }^{16}$, Tong Liu ${ }^{17}$, Roberto Furlanetto Júnio $^{18}$, Herney Andrés García-Perdomo ${ }^{19}$ and Giuseppe Biondi-Zoccai ${ }^{20}$

${ }^{1}$ Federal University of Uberlândia, Department of Clinical Research, Brazil

${ }^{2}$ Department of Clinical Research, Heart Institute (InCor), Master Institute of Education President Antonio Carlos, IMEPAC, Araguari, Brazil

${ }^{3}$ HCFMUSP- University of São Paulo Medical School, Department of Cardiology, São Paulo, Brazil

${ }^{4}$ Faculty of Medicine ABC, Department of Cardiology Santo André, Brazil

${ }^{5}$ Cardiovascular Research Center, ShahidSadoughi University of Medical Sciences, Department of Cardiology, Yazd, Iran

${ }^{6}$ Department of Specialized and General Surgery, Fluminense Federal University, Rio de Janeiro, Brazil

${ }^{7}$ Dante Pazzanese Institute of Cardiology,Department of Clinical Research, São Paulo, Brazil

${ }^{8}$ Graduate Program in Medicine and Health, Department of Health and Sciences, Federal University of Bahia, Brazil

${ }^{9}$ Federal University of Minas Gerais, Department of Cardiology, MG, Brazil

${ }^{10} \mathrm{Federal}$ University of Mato Grosso, Department of Medicine, MT, Brazil

${ }^{11}$ FOP Unicamp, Department of Clinical Research, SP, Brazil

${ }^{12}$ Division of Cardiology, Duke University Medical Center, Department of Clinical Research, Durham, NC, USA 13Monash Cardiovascular Research Centre and

Monash Heart, Department of Cardiology, Clayton, Victoria, Australia

${ }^{14}$ Tehran University of Medical Sciences, Department of Medicine, Iran

${ }^{15}$ University of Connecticut/Hartford Hospital Evidence-Based Practice Center, Hartford, Department of Comparative Effectiveness and Outcomes Research Health Outcomes, CT, USA

${ }^{16}$ Department of Medicine and Therapeutics and Li Ka Shing Institute of Health Sciences, Faculty of Medicine, Chinese University of Hong Kong, Hong Kong, China ${ }^{17}$ Tianjin Key Laboratory of Ionic-Molecular Function of Cardiovascular Disease, Department of Cardiology, Tianjin Institute of Cardiology, the Second Hos-pital of Tianjin Medical University, Tianjin, China

${ }^{18}$ Exercise Biology Research Group (BioEx), Health Sciences Institute, Universidade Federal do Triângulo Mineiro (UFTM), Uberaba, Minas Gerais, Brazil

${ }^{19}$ School of Medicine, Universidad del Valle, Cali, Colombia and UROGIV Research Group, Universidad del Valle, Cali, Colombia

${ }^{20}$ Department of Angio-CardioNeurology, IRCCS Neuromed, Pozzilli, Department of Medico-Surgical Sciences and Biotechnologies, Sapienza University of Rome, Latina, Italy

\begin{abstract}
Introduction: Patients with peripheral artery disease (PAD) have been found to suffer from diabetes, obesity, lipid abnormalities, including elevated levels of total and LDL-cholesterol as well as triglyceride levels. Epicardial adipose tissue (EAT) is a metabolically active fat depot, abundant in proinflammatory cytokines, and has been correlated with the extent and severity of PAD.The aim of this study is to examine this association of EATwith PADin different ages and sex.

Methods and analysis: We will search the PubMed, EMBASE, Cochrane Library, Web of Science, Embase, google scholar for unpublished or undergoing research listed in registry platforms. The update systematic review and meta-analysis will be conducted using published studies that will be identified from electronic databases (ie, PubMed, EMBASE, Web of Science, and Google Scholar. Studies that (1) examined the association betweenEAT and PAD, (2) focus on cohort, case-control and cross-sectional studies, (3) wereconducted among in adults aged 40 to 70 years., (4) provided sufficient data for calculating ORs or relative risk with a $95 \%$ CI, (5) were published as original articles written in English or other languages, and (6) have been published until January 2018 will be included. Study selection, data collection, quality assessment and statistical syntheses will be conducted based on discussions among investigators.
\end{abstract}

Ethics and dissemination: Ethics approval was not required for this study because it was based on published studies. The results and findings of this study will be submitted and published in a scientific peer-reviewed journal.

Trial registration number: PROSPERO (CRD42018083417).

${ }^{*}$ Correspondence to: Leonardo Roever, Department of Clinical Research, Federal University of Uberlândia, Heart Institute (InCor), Master Institute of Education President Antonio Carlos, IMEPAC, Araguari, Brazil, E-mail: leonardoroever@hotmail.com

Key words: epicardial fat, peripheral artery disease, systematic review

Received: August 16, 2018; Accepted: August 28, 2018; Published: August 31, 2018 
Abbreviations: $\mathrm{CIs}=$ Confidence Intervals, $\mathrm{HDL}=$ high-density lipoprotein, $\mathrm{LDL}=$ low-density lipoprotein, $\mathrm{MD}=$ mean difference, $\mathrm{RR}=$ risk ratio, $\mathrm{WC}=$ waist circumference, $\mathrm{PAD}=$ peripheral artery disease, $\mathrm{EAT}=$ epicardial adipose tissue

\section{Strengths and limitations of this study}

This systematic review and meta-analysis will offer better understanding regarding the association between metabolic syndrome and PAD.

The findings from this study will be useful for assessing of EAT and the risk factors in PAD and determining approaches for prevention of PAD the future.

An improved understanding of this relationship may help to inform public health PAD prevention strategies.

Included studies may have substantially different methodologies, which could limit our ability to draw reliable conclusions from the existing evidence base. Depending on the results, confounding factors that were not adjusted for in the selected studies and low generalizability can be limitations. Individual patient data will not be available.

\section{Background}

It is estimated that approximately $22 \%$ of patients with PAD will eventually die from cardiovascular events and therefore aggressive management of cardiovascular risk factors is a primary focus of treatment [1-7].

Although the literature on the EAT and the risk factors for PAD has been increasing, to our knowledge, a systematic review of the association between EAT and risk of PAD has not yet been conducted [1-9].

This study aims to systematically assess the association between EAT and PAD in adults aged 34 to 70 years; and to provide a framework to further understand these factors in order to better target prevention strategies.

\section{Methods/design}

This systematic review of the literature will follow the Preferred Reporting Items for Systematic Reviews and Meta-Analyses (PRISMA) recommendations. The databases PubMed, Embase, Web of Science, Google scholar, and Cochrane were searched for articles. Our search will focus on cohort, case-control and cross-sectional studies examining the association between EAT and PAD. The primary outcome is PAD. Two reviewers will independently screen articles, extract relevant data and assess the quality of the studies.

The objective of this systematic review and meta-analysis was to explore the association between EAT and the presence of PAD. The secondary aims were to evaluate whether increasing EAT volume is associated with $\mathrm{PAD}$ presence and the strength of association with presence of PAD by EAT measurement method in PAD might additional risk factor and predictor of CVD events, and mortality (CVmortality, mortality and all-cause mortality) [1-2].

The study is registered with PROSPERO (CRD42018083417). This protocol conforms to the Preferred Reporting Items for Systematic Reviews and Meta-Analyses Protocols (PRISMA-P) guidelines [10-14].

\section{Systematic review registration}

This protocol is registered in the PROSPERO registry of the University of YorkReference number :(CRD42018083417).

\section{Objectives}

The primary objective is to identify and summarize the associated with EAT diagnosis and with PAD in adults (34-70 years) in different ages and sex.

\section{Eligibility criteria}

The PICOS strategy (population, intervention (changed to exposure for the purposes of this review of observational studies), comparator, outcome, study characteristics was used to define the eligibility criteria for this study:

Inclusion criteria: Cohort, case-control and cross-sectional studies. Adult patients with and without PAD who underwent imaging to measure epicardial fat.

Exclusion criteria: Studies with overlapping data.

Data items on the following five domains will be extracted:

1. Population: characteristics of the study population (eg, mean/ median age, ethnic distribution), inclusion and exclusion criteria

2. Exposure: definition and identification of EAT. Both criteria must be met:

- Measurement of epicardial adipose tissue (EAT) by linear or volumetric analysis using MRI, CT or echocardiography.

- Patients who have undergone a full 3D assessment of the myocardium in order to obtain full volumetric measurement of the epicardial adipose tissue. This can only be performed with MRI or CT imaging. Echocardiography as an imaging modality to obtain myocardial function, specifically measures of systolic function (e.g. ejection fraction), diastolic function (e', E/A ratio and other Doppler derived indices, as well as global longitudinal strain analysis), or full volume cardiac CT or MRI imaging to obtain ventricular volumes function parameters.

- Comparators: definition and identification of unexposed individuals, number of unexposed subjects

- Outcomes: definition and identification of primary (CVD outcomes, and death (CV mortality, all-cause mortality) and secondary outcomes (changes in pain free walking distance (PFWD) and quality of life (QOL)).

- Study characteristics: authors, publication year, setting/source of participants, design, methods of recruitment and sampling, period of study, length of follow-up time (if relevant), aims and objectives.

\section{Outcomes}

\section{Primary outcomes}

Patients with a diagnosis of EAT that present CVD outcomes, and death (CV mortality, all-cause mortality).

\section{Secondary outcomes}

The association of EAT with PAD, by EAT measurement method.

The secondary outcome measures include changes in pain free walking distance (PFWD) and quality of life (QOL).

For studies meeting the inclusion criteria, we will additionally assess the following outcomes: TIA (a transient episode of neurological dysfunction caused by focal brain, spinal cord or retinal ischaemia without acute infarction) and subtypes of stroke (ischaemic vs 


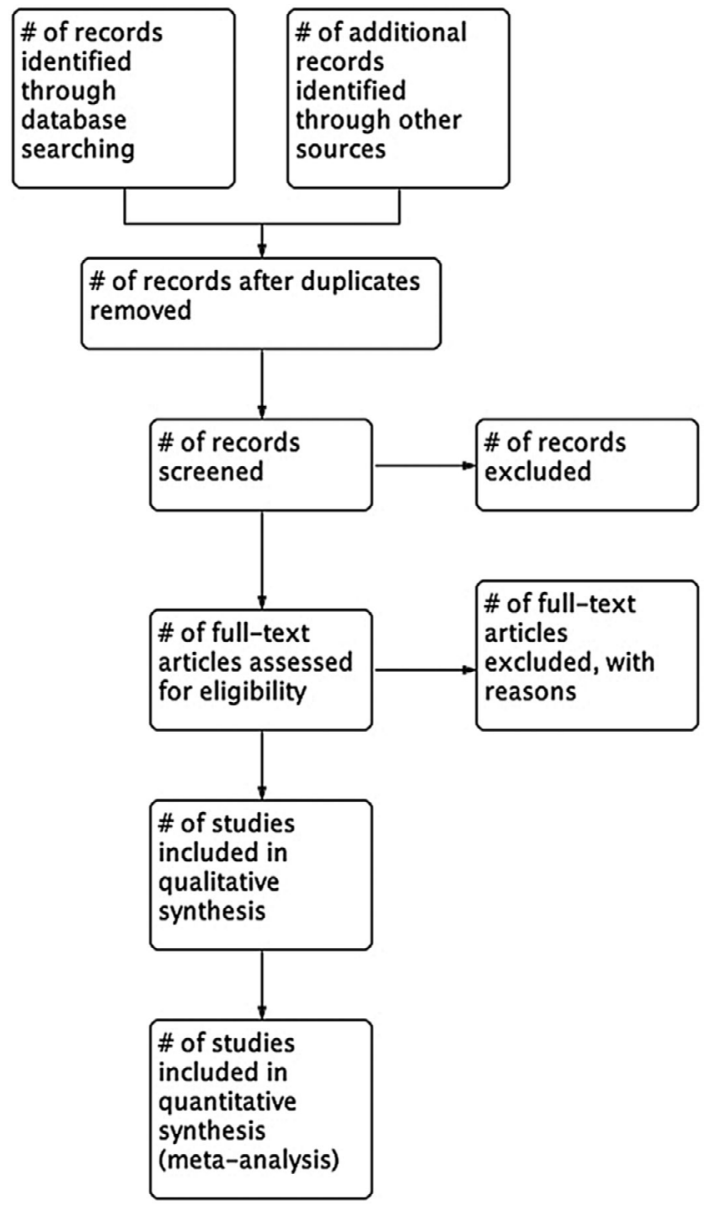

Figure 1. Flow diagram of study selection process

haemorrhagic). Most strokes (approximately 85\%) are ischaemic (an episode of neurological dysfunction caused by focal, cerebral, spinal or retinal infarction), compared with haemorrhagic (neurological dysfunction caused by a focal collection of blood within or on the surface of the brain). Eligibility criteria may be further developed, in an iterative process, after preliminary searches.

\section{Study design}

This is a systematic review and meta-analysis protocol of prospective cohort studies, following the PRISMA-P (Preferred Reporting Items for Systematic Reviews and Meta-Analysis protocols) guideline [14]. The systematic review and meta-analysis will be reported according to the PRISMA (Preferred Reporting Items for Systematic Reviews and Meta-Analyses) guideline [15]. The whole process of study selection is summarized in the PRISMA flow diagram (Figure 1).

\section{Search strategy}

A systematic review of the literature will be conducted. A language restriction shall not be applied to the search. If there are relevant nonEnglish abstracts, attempts shall be made to translate them wherever possible. The following bibliographic databases (Embase, PubMedMEDLINE, Web of Science, Cochrane Library, and Google Scholar) will be searched for articles published until January 2018. Our search focuses on studies examining the association between EAT and PAD risk in adults (34-70 years [11]. At each step of the selection process, reasons for inclusion/exclusion will be recorded in the PRISMA Flowchart [13].

\section{Data collection}

A record will be kept of all searches and search decisions to ensure reproducibility. Search results will be exported to a citation management program (EndNote ver. 7.0). Duplicates will be removed and retained separately. The resulting references will be exported separately to the two reviewers for independent review using Covidence.

\section{Selection of studies}

Two authors (LR, FCV) will independently screen all titles and abstracts identified through the literature searches and will exclude all records clearly not meeting inclusion criteria. Disagreements will be resolved by consensus. The selection process will be pilot tested to ensure a high degree of agreement between reviewers. Full text of the remaining studies will then be retrieved. The same two authors (LR, FCV) will independently assess the papers for fulfilment of inclusion criteria. In case of differences of opinion regarding study inclusion, a third review author (GBZ) will serve as arbiter. To avoid double counting, if multiple publications based on the same cohort of participants are retrieved, only the study reporting the largest sample size will be used. The reasons for excluding papers for which the full text was retrieved will be documented.

\section{Data extraction and management}

A data extraction form will be used to collect details from the included studies. The form includes information on study design, patient population, and presence of PAD. Two review authors (LR and 
FCV) will independently extract the data. The data extraction form will be pilot tested on several papers to ensure consistency and that all relevant information is being captured. If necessary, a statistician will review the extraction of data to further ensure quality and reliability. Authors will be contacted for missing data.

Data will be extracted using a standardized template. We will use the PICOS (Population, Intervention, Comparator, Outcomes and Study design) framework, originally devised to formulate a research question, as a basis to develop data extraction criteria. As this is an aetiological study, 'exposure' will replace 'intervention' and 'study characteristics' will replace 'study design'.

In terms of the study results, unadjusted and fully adjusted effect estimates for the association between low EAT and PAD will be recorded. Details of the confounders measured and adjusted for will also be noted. Results of any additional stratified analyses will also be recorded. Where possible, results from additional subgroup analyses with evidence regarding our non-primary objectives will also be recorded, for example, the association between low EAT and the secondary outcomes (Stroke subtype or TIA).

\section{Assessment of methodological quality}

Two investigators (LR and FCV) will independently assess each selected study for study quality using the Newcastle-Ottawa Quality Assessment Scale (NOS) [16] The NOS evaluates cohort studies based on eight items categorized into the following three groups: (1) selection of the study cases, (2) comparability of the population, and (3) ascertainment of whether the exposure or outcome includes any risk of bias (i.e., selection bias or bias from lost to follow-up). The NOS is scored ranging from 0 to 9 , and studies with scores $\geq 7$ are considered as high quality.16 Discrepancy of quality assessment among the investigators will be solved by discussion and consensus among all authors.

\section{Data synthesis and statistical analysis}

We anticipate that there may be significant heterogeneity in the prevalence of EAT features of PAD. There are several factors that could contribute to such heterogeneity. The relative risk (RR), and odds ratio (OR) are the way the result will be expressed statistically.

These factors include the following: differences in demographic and clinical features (e.g., age, hypertension, renal disease, smoking, duration and severity of diabetes) among study cohorts; differences in definitions of EAT. An I2 statistic will be calculated for the studies to be included in each proposed meta-analysis (i.e. for each neuroradiology correlate of interest) with values of 25,50 , and $75 \%$ suggesting low, moderate, or high degrees of heterogeneity, respectively, which report a dichotomized (i.e., present or absent) or categorical (i.e., absent, mild, moderate, severe) shall be harmonized for meta-analysis if deemed appropriate by our statistician. Other types of rating scales shall not be included in a meta-analysis and the data based on any such data scale would be presented in narrative form.

If significant heterogeneity between studies, as determined by consultation with our statistician, prevents meaningful pooling of the data, we will limit ourselves to providing a narrative description of observed trends. Given the heterogeneity of the populations studied, assumption of a fixed effect size across populations would not be justified, thus analyses would be performed using a random effects model. Given the dichotomized (presence or absence) or categorical (severity measure) nature of our data of, meta-analysis will be performed a random effects analysis. We will also add funnel graphs, publication bias analysis and a meta-regression analysis.

If there are sufficient data to allow such analyses (in principle from as few as a single high-quality study, but if possible by pooling data from multiple studies), we will perform subgroup analyses for participants with renal disease and participants with hypertension. In addition, if sufficient data are available, we shall perform subgroup analyses by age and diabetes duration. Funding sources and conflict of interest will be extracted from included studies. Statistical analysis will be performed using RevMan software.

\section{Strategy for data synthesis}

The data of interest presented as continuous (mean value and $\mathrm{SD}$ ) will be used to perform meta-analysis to obtain the standardized mean difference (SMD) and 95\% confidence interval (CI). Cocharn's Q-statistic and I-squared test will be used to test for heterogeneity between the included studies. If an I-squared value will be greater than $50 \%$ or a $p$ value of the $Q$-test will be less than 0.05 , indicating maximal heterogeneity among the included studies, a random-effect model will be put into use.

\section{Analysis of subgroups or subsets}

The subgroup meta-analyses will be conduct according to the prespecified study-level characteristics using a fixed-effects meta-analysis and if there is substantial heterogeneity, we will use the random effects model. The sources included location, sex, age, method of EAT assessment, the definition of PAD. We also will conduct sensitivity analyses to evaluate the potential sources of heterogeneity in the analyses.

\section{Summary of Evidence}

We will produce a narrative synthesis of the main results extracted from articles in full text. A summary of the included studies will provide information on the authors, study design, participants, number and age of the subjects, theoretical structure (if relevant), alcohol consumption (as primary outcome of interest), main findings, Study information. Special emphasis will be placed on the identification of EAT and the risk of PAD. In the presentation of the results, we will try to separate the factors for which the evidence of causality is strong (from longitudinal studies) and factors for which the causal nature of the relationship is less secure (cross-sectional data). A graphical summary of all the data they represent will be provided and take into account the number of studies that provide evidence of a factor and the relative strength of the association presented based on study design and quality assessment. The membership level will be evaluated based on adjusted data.

\section{Discussion}

This systematic review will synthesize research evidence to establish whether the risk of developing PAD is relatively high in adults with EAT. Strengths and limitations will be highlighted in the identified evidence. Strength of observational data may include large sample size, high rate of follow-up and frequency of PAD more likely to be representative of the population at risk. Limitations may include the quality of data extracted which may not allow studies to be combined in a meta-analysis. This may be overcome by presenting the findings in a descriptive manner. This review will be conducted in collaboration with an experienced librarian who helped appraise the search criteria, refine the keywords and MeSH terms and identify appropriate database(s). To the best of our knowledge, no reviews have been published exploring 
the study question; however, if a review addressing a similar question is published, it will be incorporated in this review and added in a metaanalysis if feasible.

\section{Implications of results}

This systematic review will provide an updated and quantifiable estimate of the risk of PAD in adults with EAT. Furthermore, the systematic search will identify where future research is required. For instance, this review may inform a prognostic study which may be useful in understanding the course and factors associated with PAD development.

\section{Amendments}

If it is necessary, we will update this protocol in the future. We will submit the original protocol, final protocol and summary of changes as a supplement.

\section{Ethics and dissemination}

\section{Ethical issues}

No ethical approval is required because this study includes no confidential personal data or interventions with the patients.

\section{Publication plan}

The procedures of this systematic review and NAM will be conducted in accordance with the PRISMA-compliant guideline. The results of this systematic review and NAM will be submitted to a peerreviewed journal for publication.

\section{Authors' information}

Not applicable.

\section{Competing interests}

The authors declare that they have no competing interests.

\section{Consent for publication}

Not applicable.

\section{Ethics approval and consent to participate}

Not applicable.

\section{Availability of supporting data \\ Not applicable.}

\section{Funding}

Not applicable.

\section{Authors' contributions}

LR, ASRB, ALDD, ACF, NPS, PMMD, RMLS, JLO, MN, AD, PARD, FRS, GBFO, RFJ, GBZ, SAH, PEOR, AJG, RMP, ACF, PMMD, TMF, NN, SA, CD, PFSG, A.A, AVH, MIR, RDL and FCV conceived the study idea and devised the study methodology. LR, ASRB, ACPC and ESR participated in the design and coordination of the study. LR was primarily responsible for protocol writing and developed the search strategy. LR and FCV will screen identified literature, conduct data extraction and analyses the review findings. All authors read the drafts, provided comments and agreed on the final version of the manuscript.

\section{Acknowledgements}

Not applicable.

\section{References}

1. Smilowitz NR, Gupta N, Guo Y, Beckman JA, Bangalore S, et al. (2018) Trends in cardiovascular risk factor and disease prevalence in patients undergoing non-cardiac surgery. Heart 104: 1180-1186. [Crossref]

2. Oliveira GB, Avezum A, Roever L (2015) Cardiovascular disease burden: evolving knowledge of risk factors in myocardial infarction and stroke through population-based research and perspectives in global prevention. Front Cardiovasc Med 2: 32. [Crossref]

3. Basurto Acevedo L, Barrera Hernández S, Fernández Muñoz MJ, Saucedo García RP, Rodríguez Luna AK, et al. (2018) An increase in epicardial fat in women is associated with thrombotic risk. Clin Investig Arterioscler 30: 112-117. [Crossref]

4. Roever LS, Resende ES, Diniz AL, Penha-Silva N, Veloso FC, et al. (2016) Abdominal obesity and association with atherosclerosis risk factors. Medicine (Baltimore) 95: e1357. [Crossref]

5. Roever L, Resende ES, Diniz AL, Penha-Silva N, Veloso FC, et al. (2015) Ectopic adiposopathy and association with cardiovascular disease risk factors: The Uberlândia Heart Study. Int J Cardiol 190: 140-142. [Crossref]

6. Roever L, Resende ES, Veloso FC, Diniz AL, Penha-Silva N, et al. (2015) Perirenal fat and association with metabolic risk factors. Medicine (Baltimore) 94: e1105. [Crossref]

7. van Rooy MJ, Pretorius E (2015) Metabolic syndrome, platelet activation and the development of transient ischemic attack or thromboembolic stroke. Thromb Res 135: 434-442. [Crossref]

8. Sarrafzadegan N, Gharipour M, Sadeghi M, Nezafati P, Talaie M, et al. (2017) Metabolic Syndrome and the Risk of Ischemic Stroke. J Stroke Cerebrovasc Dis 26 286-294. [Crossref]

9. Sánchez-Iñigo L, Navarro-González D, Fernández-Montero A, Pastrana-Delgado J, Martínez JA (2017) Risk of incident ischemic stroke according to the metabolic health and obesity states in the Vascular-Metabolic CUN cohort. Int J Stroke 12: 187-191. [Crossref]

10. Chei CL, Yamagishi K, Tanigawa T, Kitamura A, Imano H, et al. (2008) Metabolic Syndrome and the Risk of Ischemic Heart Disease and Stroke among Middle-Aged Japanese. Hypertens Res 31: 1887-1889.

11. Fang X, Liu H, Zhang X, Zhang H, Qin X, et al. (2016) Metabolic Syndrome, Its Components, and Diabetes on 5-Year Risk of RecurrentStroke among Mild-to-Moderate Ischemic Stroke Survivors: A Multiclinic Registry Study. J Stroke Cerebrovasc Dis 25: 626-634. [Crossref]

12. Glasziou P, Irwig L, Bain C, Colditz G (2001) Systematic reviews in health care: practical guide. Cambridge: Cambridge University Press.

13. Newcastle Ottawa Scale (2017) The Newcastle-Ottawa Scale (NOS) for assessing the quality of nonrandomised studies in meta-analyses.

14. Moher D, Liberati A, Tetzlaff J, Altman DG, PRISMA Group (2009) Preferred reporting items for systematic reviews and meta-analyses: the PRISMA statement. Ann Intern Med 151: 264-269. [Crossref]

15. Shamseer L, Moher D, Clarke M, Ghersi D, Liberati A, et al. (2015) Preferred reporting items for systematic review and meta-analysis protocols (PRISMA-P) 2015 elaboration and explanation. BMJ 349: g7647.

16. Wells GA, Shea B, O'Connell D. The Newcastle-Ottawa Scale (NOS) for assessing the quality of nonrandomized studies in meta-analyses. Secondary The Newcastle-Ottawa Scale (NOS) for assessing the quality of nonrandomized studies in met analyses.

Copyright: (C2018 Roever L. This is an open-access article distributed under the terms of the Creative Commons Attribution License, which permits unrestricted use, distribution, and reproduction in any medium, provided the original author and source are credited. 